\title{
Use of fibrinolytic agents in the management of complicated parapneumonic effusions and empyemas
}

\author{
Steven A Sahn
}

Division of Pulmonary and Critical Care Medicine, Allergy and Clinical Immunology, Medical University of South Carolina, Charleston,

South Carolina 29425-2220, USA

Introductory article

Randomised controlled trial of intrapleural streptokinase in community acquired pleural infection

RJO Davies, ZC Traill, FV Gleeson

Background. Standard treatment for pleural infection includes catheter drainage and antibiotics. Tube drainage often fails if the fluid is loculated by fibrinous adhesions when surgical drainage is needed. Streptokinase may aid the process of pleural drainage, but there have been no controlled trials to assess its efficacy. Methods. Twenty four patients with infected community acquired parapneumonic effusions were studied. All had either frankly purulent/culture or Gram stain positive pleural fluid (13 cases; $54 \%$ ) or fluid which fulfilled the biochemical criteria for pleural infection. Fluid was drained with a $14 \mathrm{~F}$ catheter. The antibiotics used were cefuroxime and metronidazole or were guided by culture. Subjects were randomly assigned to receive intrapleural streptokinase, $250000 \mathrm{IU}$ daily, or control saline flushes for three days. The primary end points related to the efficacy of pleural drainage - namely, the volume of pleural fluid drained and the chest radiographic response to treatment. Other end points were the number of pleural procedures needed and blood indices of inflammation. Results. The streptokinase group drained more pleural fluid both during the days of streptokinase/control treatment (mean (SD) 391 (200) m/ versus 124 (44) ml; difference $267 \mathrm{ml}$, 95\% confidence interval (Cl) 144 to 390; $p<0.001)$ and overall (2564 (1663) $\mathrm{ml}$ versus 1059 (502) $\mathrm{ml}$; difference $1505 \mathrm{ml}$, 95\% Cl 465 to 2545; $p<0.01)$. They showed greater improvement on the chest radiograph at discharge, measured as the fall in the maximum dimension of the pleural collection $(6.0(2.7) \mathrm{cm}$ versus $3.4(2.7) \mathrm{cm}$; difference $2.9 \mathrm{~cm}$, $95 \% \mathrm{Cl} 0.3$ to $4.4 ; p<0.05)$ and the overall reduction in pleural fluid collection size $(p<0.05$, two tailed Fisher's exact test). Systemic fibrinolysis and bleeding complications did not occur. Surgery was required by three control patients but none in the streptokinase group. Conclusions. Intrapleural streptokinase probably aids the treatment of pleural infections by improving pleural drainage without causing systemic fibrinolysis or local haemorrhage. (Thorax 1997;52:416-421)

Scope of the problem

Pneumonia, one of the most common community acquired and nosocomial infections, is associated with a high incidence of pleural effusions. ${ }^{1}$ The $36-57 \%$ incidence of parapneumonic effusions translates into approximately one million persons per year in the United States with effusions associated with pneumonia..$^{2-4}$ These parapneumonic effusions usually resolve spontaneously if patients are treated with appropriate antibiotics shortly after the initial symptoms of pneumonia. However, a small percentage of parapneumonic effu- sions will become complicated, either loculated nonpurulent fluid or an empyema (frank pus). Empyema thoracis has a high likelihood to occur in individuals who delay seeking medical attention and those with co-morbid conditions such as alcoholism or chronic lung disease. ${ }^{4}$ In a few cases complicated parapneumonic effusions are caused by delayed or inappropriate treatment by the physician. ${ }^{5}$

The natural history of a complicated parapneumonic effusion is to develop a single loculus or multiple loculations and to progress to an empyema cavity. Em- 
pyema, from the Greek meaning accumulation of pus in a body cavity, represents the end stage of a complicated parapneumonic effusion. The optimal approach to the diagnosis and management of complicated parapneumonic effusions is controversial because of the pneumonic . The availability of new options for management of these effusions further confounds clinical decision making. ${ }^{7}$ A 1992 interactive session of an American College of Chest Physicians' meeting on management of pleural space infections showed non-uniformity of opinions on treatment.

\section{Pathophysiology of parapneumonic effusions}

The clinical features of the patient with a parapneumonic effusion depends on the stage of the effusion at presentation and can vary from symptoms of pneumonia only with a small, free flowing, non-purulent, non-infected effusion to severe chest pain, high fever and systemic symptoms with a classic empyema. The stage of the effusion at presentation correlates directly with the time interval between the onset of pneumonia and physician contact.

A parapneumonic effusion is formed when permeability pulmonary oedema fluid moves into the pleural space at a rate exceeding the lymphatic drainage capacity of the parietal pleura. The increased production of pleural fluid results from neutrophil induced endothelial injury of the parenchymal, subpleural and pleural vessels. The proteinaceous fluid that moves from the intravascular to extravascular space increases the parintravascular to extravascular space increases the parvisceral pleural mesothelial cells into the pleural space. Presumably in most pneumonias pleural fluid production increases, but the excess fluid produced is removed rapidly by normal functioning parietal pleural lymphatics not allowing a clinical pleural effusion to form. It is probable that only when the parietal pleural form. It is probation of mesothelial cell swelling or fibrin occlusion does fluid of mesothelial cell swelling or fibrin occlusion does fluid
accumulate to a degree that allows clinical detection. accumulate to a degree that allows clinical detection. The earliest parapneumonic effusion, typical of the exudative (capillary leak) stage, is characterised clinically by an ipsilateral, small, free flowing, turbid exuche a moderate number of neutrophils. The biochemistry and not pleural space infection. Therefore, the pleural and not pleural space infection. Therefore, the pleura
fluid $\mathrm{pH}$ is typically $>7.30$, the glucose level is $>60 \mathrm{mg} /$ fluid $\mathrm{pH}$ is typically $>7.30$, the glucose level is $>60 \mathrm{mg} /$
$\mathrm{dl}$, and the lactate dehydrogenase (LDH) concentration $\mathrm{dl}$, and the lactate dehydrogenase (LDH) concentration
is $<500 \mathrm{IU} / \mathrm{I}^{21011}$ If the pneumonia is treated with appropriate antibiotics at this stage the effusion is unlikely to progress to the second or fibrinopurulent stage.

However, if the pneumonia remains untreated, most commonly due to delayed patient presentation, endothelial injury becomes more severe and widespread and a larger volume of pleural fluid is formed. Without the inhibition of antimicrobial agents, bacterial multiplication in the lung is unchecked and bacteria move from the lung interstitium into the pleural space and eventually overwhelm the capacity of the intrapleura phagocytes (neutrophils and macrophages) and parietal pleural lymphatics and become persistent, resulting in a positive pleural fluid Gram stain and culture. This fibrinopurulent (bacterial invasion) stage typically ocfibrinopurulent (bacterial invasion) stage typically oc-
curs several days following the initial pleural fluid for-
mation and is characterized by an absolute increase in mation and is characterized by an absolute increase in
pleural fluid neutrophils, which have an increased burst in metabolic activity during phagocytosis, resulting in increased glucose utilisation and increased production of glucose end products, carbon dioxide and lactic acid, ultimately leading to cell death. These pathophysiological changes result in pleural fluid acidosis, a low glucose concentration, and an increased LDH concentration. ${ }^{1213}$ Thus, the typical pleural fluid triad of the fibrinopurulent stage of a parapneumonic effusion is a $\mathrm{pH}$ of $<7.20$, a glucose level of $<40 \mathrm{mg} / \mathrm{dl}$, and an is a $\mathrm{pH}$ of $<7.20$, a glucose level of $<40 \mathrm{mg} / \mathrm{dl}$, and an $\mathrm{LDH}$ level of $>1000 \mathrm{IU} / \mathrm{l}^{21011}$ In addition, pleural fluid becomes clottable as plasma proteins leak into the pleural space in conjunction with a loss of the fibrinolytic activity of the inflamed pleura resulting in pleural fibrin deposition. ${ }^{1415}$ Fibroblasts migrate from the sub-mesothelial connective tissue into the pleural fluid, unimpeded by the injured mesothelial cell barrier. The secretion of glycosaminoglycan and collagen, in concert with fibrin deposition, compartmentalise the fluid into loculations by bridging the two pleural surfaces and limiting lung expansion. ${ }^{15}$ At some unknown point during the fibrinopurulent stage the clinician loses the ability to treat the patient with antibiotics alone and pleural space drainage becomes necessary to prevent pleural sepsis.

The natural progression of the parapneumonic effusion leads to the final stage, the organisational or empyema stage, over a period of at least two weeks to several weeks that results in the formation of either a single thick walled cavity or multiple loculations due to the continued fibroblast migration and growth into the coagulable pleural fluid matrix. An inelastic pleural "peel" inhibits pleural space drainage as well as lung "peep" in this stage is classic empyema fluid which is pus (a thick, whitish-yellow, opaque coagulum) that always requires drainage. Pus assumes its specific character because of coagulability of pleural fluid, the abundance of cellular debris, and fibrin and collagen deposition. Bacteria tend to persist in empyema fluid because of the decreased bacterial opsonisation from complement depletion and pleural fluid acidosis ${ }^{16}$ An empyema spontaneously; it may herald its presence by draining through the chest wall (empyema necessitatis) or into the lung (bronchopleural fistula).

Pleural fluid in the exudative stage does not need drainage while an empyema always needs to be evacuated. The real dilemma facing the clinician is how best to manage the patient in the fibrinopurulent stage of a antibiotics alone, while others require pleural space drainage which can be accomplished by several methods including serial therapeutic thoracenteses, standard chest tubes, or image guided small bore catheters with or without fibrinolytic agents, empyectomy and decortication by thoracoscopy or standard thoracotomy, or open drainage.

\section{Features that suggest the need for pleural space} drainage

The rapid identification of patients likely to develop complicated parapneumonic effusions should improve clinical outcome by allowing early pleural space drainage. It is unlikely that common clinical parameters such peak temperature, presence or absence of pleuritic chest pain, or number of lobes involved with pneumonia can differentiate between those parapneumonic effusions that would benefit from pleural space drainage and those that can be treated with antibiotics alone. ${ }^{217}$

There are, however, clinical features, chest radiographic, ultrasound, and chest CT findings, and pleural fluid characteristics that suggest that a parapneumonic 
effusion has a high likelihood of requiring drainage. The clinical features that suggest the need for pleural space drainage include prolonged symptoms prior to presentation, anaerobic infection, ${ }^{18}$ the combination of leucocytosis, anaemia and hypoalbuminaemia ${ }^{19}$ failure of clinical response to antibiotic therapy, ${ }^{20}$ and virulence of the pathogen. ${ }^{42122}$ Chest radiographic and CT findings that suggest the need for pleural space drainage include an effusion of $>40 \%$ of the hemithorax,${ }^{19}$ the presence of an air fluid level, ${ }^{18}$ loculation, ${ }^{211} 1723$ multiple loculations, the size of the loculations, pleural enhancement or pleural thickening on contrast CT scan, ${ }^{2425}$ and septations, fibrin strands, and necrotic debris ${ }^{2627}$ on ultrasonography. Pleural fluid acteristics that suggest an increased likelihood that a parapneumonic effusion requires drainage include purulent fluid (always requires drainage), a positive Gram stain or culture, ${ }^{18}$ a low pleural fluid $\mathrm{pH}(<7.30$ or $7.20)$, low pleural fluid glucose $(<40 \mathrm{mg} / \mathrm{dl})$, and a high LDH concentration $(>1000 \mathrm{IU} / \mathrm{l}) .^{210111728-30}$

\section{Methods of pleural space drainage}

The pleural space can be drained by standard chest tubes, small bore, radiologically guided catheters (usually 8-14 F), thoracoscopic debridement, pleural irrigation and chest tube placement, standard thoracotomy with empyectomy and decortication, and open drainage with rib resection. It would be advantageous to the patient with a complicated parapneumonic effusion or empyema from the standpoint of morbidity and cost to be treated by closed drainage with eit standard chest tube or small catheter, in lieu of a more invasive surgical procedure such as thoracoscopy, standard thoracotomy, or open drainage. The major reasons for failure of tube drainage are tube malposition, tube obstruction by viscous fluid, and multiple pleura space loculations. Both tube obstruction and a multiloculated pleural space has generated interest in intrapleural fibrinolytic treatment of complicated parapneumonic effusions during the past half century.

\section{Fibrinolytic therapy}

Tillet and Sherry ${ }^{31}$ in 1949 first reported the use of fibrinolytic agents in 23 patients who had either acute fibrinous pleurisy, bacterial empyema, or haemothorax. Their patients received intrapleural instillation of both streptokinase and desoxyribonuclease that resulted in transient pleural fluid fibrinolysis and proteolysis. They also demonstrated depolymerisation of the nucleoprotein in the exudate. When toxic manifestations of the drug occurred they were limited to transient febrile the drug occurred they were limited to transient febrile
reactions and malaise. Subsequent investigations in
small numbers of patients with complicated parasmall numbers of patients with complicated parapneumonic effusions reported improvement in clinica outcome. However, the initial enthusiasm for intrapleural fibrinolytics waned because of temic adverse effects until Bergh and colleagues, ${ }^{32}$ using a purified streptokinase, reported chest radiographic improvement in 10 of 12 patients with empyemas without the need for thoracotomy and without significant adverse effects. Since that time there have been a plethora of case series using streptokinase and urokinase for initial drainage of the pleural space and for treatment of failed chest tube or small catheter drainage, obviating the need for surgical intervention.
CASE STUDIES

All studies with three or more patients using streptokinase or urokinase in the treatment of complicated parapneumonic effusions and empyemas are shown in tables 1 and 2, respectively (virtually all of the studies cited are uncontrolled case series). In general the studies show a "good success" rate in the treatment of these complicated parapneumonic effusions in the pleural complicated parapneumonic effusions in the pleural
space. However, the assessment of treatment in these space. However, the assessment of treatment in these
uncontrolled case series is largely determined by patient selection, especially the timing of the initiation of therapy to the stage of evolution of the parapneumonic effusion. As can be stated for all treatment modalities of parapneumonic effusions, the earlier that a specific treatinstituted the more likely the successful. For example, early antibiotic therapy will not only tend to prevent a parapneumonic effusion but will tend to prevent the progress from a simple uncomplicated effusion to a complicated parapneumonic effusion or empyema. Furthermore, early drainage of a free-flowing, complicated parapneumonic effusion (by biochemical parameters) will tend to prevent loculation and progression to an empyema. Likevent loculation and progression to an empyema. Like-
wise, thoracoscopy is likely to be effective if used early wise, thoracoscopy is likely to be effective if used early
in the fibrinopurulent stage of a parapneumonic effusion and less likely to be effective in a chronic empyema. Fibrinolytic therapy is also more likely to be successful early in the fibrinopurulent stage of parapneumonic effusion and unlikely to be successful in an organised empyema cavity when mature fibrin adhesions and a empyema cavity when mature fibrin
visceral pleural peel have developed.

The key to successful fibrinolytic drainage of complicated parapneumonic effusions is correct placement of tubes or catheters with radiological guidance early in the evolution of the parapneumonic effusion, followed by frequent monitoring (more than once daily) of tube placement and fibrinolytic effectiveness by assessing the volume of tube drainage and immediate re-insillation of the fibrinolytic agent if necessary. In the properly selected patient, attention to detail using a strict protocol
will be critical in determining a successful outcome.

RANDOMISED CONTROLLED AND COMPARATIVE STUDIES There has only been one randomised controlled trial evaluating an intrapleural fibrinolytic in the management of complicated parapneumonic effusions which forms the index article of this review. In this study Davies and associates ${ }^{47}$ studied 24 patients with community acquired pneumonia and parapneumonic effusions (13 of the 24 patients had either frank pus or culture or Gram stain positivity (empyema), while the other 11 cases had a pleural fluid $\mathrm{pH}$ of $<7.10, \mathrm{LDH}$ concentration of $>1000$ IU/1, and pleural fluid/blood glucose cetiof $<0.25$ with loculation or septation of ple of pleural fluid on chest CT scanning and ultrasonography, respectively (complicated parapneumonic); tables 1 and 3). Pleural fluid was drained with a $14 \mathrm{~F}$ catheter and antibiotics were given as a standard antibiotic regimen or guided by culture. Twelve patients were randomised to receive intrapleural streptokinase, $250000 \mathrm{U}$ daily, and 12 patients were randomised to receir ent that the streptokinase group drained more pleural fluid both during the three days of treatment and during the overall drainage period. In addition, the streptokinase group showed a greater improvement on the chest radiograph and overall reduction in pleural fluid collection size at discharge. There was no biochemical or clinical evidence of systemic fibrinolysis. Surgery was required in three 
Table 1 Intrapleural streptokinase in the treatment of complicated parapneumonic effusions (CPE) and pneumonic empyemas

\begin{tabular}{|c|c|c|c|c|c|c|c|}
\hline Reference & Study design & $\begin{array}{l}\text { Number of } \\
\text { patients/disease }\end{array}$ & $\begin{array}{l}\text { Streptokinase dose } \\
(U)\end{array}$ & Success criteria & $\begin{array}{l}\text { Success } \\
\text { rate (\%) }\end{array}$ & Complications & Comments \\
\hline $\begin{array}{l}\text { Bergh } \\
(1977)^{32}\end{array}$ & $\begin{array}{l}\text { Retrospective } \\
\text { case series }\end{array}$ & 12 empyemas & $\begin{array}{l}250000 \text { in } 100 \mathrm{ml} \\
\text { NS daily; clamped } \\
4 \mathrm{~h}\end{array}$ & $\begin{array}{l}\text { Volume of fluid } \\
\text { drainage; CXR } \\
\text { improvement }\end{array}$ & 83 & $\begin{array}{l}1 \text { fever } \\
1 \text { decreased } \\
\text { Hgb }\end{array}$ & $\begin{array}{l}\text { Failed conventional } \\
\text { drainage; chest tubes } \\
10-12 \mathrm{~mm} ; 2-10 \\
\text { inntillatios }\end{array}$ \\
\hline $\begin{array}{l}\text { Lysy } \\
(1989)^{33}\end{array}$ & $\begin{array}{l}\text { Retrospective } \\
\text { case series }\end{array}$ & 3 empyemas & $\begin{array}{l}250000 \text { in } 100 \mathrm{ml} \\
\text { NS daily; clamped } \\
4 \mathrm{~h}\end{array}$ & $\begin{array}{l}\text { Clinical and CXR } \\
\text { improvement }\end{array}$ & 100 & None & $\begin{array}{l}\text { Instlllations } \\
\text { Failed chest tube } \\
\text { drainage; } 4-10 \\
\text { instillations }\end{array}$ \\
\hline $\begin{array}{l}\text { Mitchell } \\
(1989)^{34}\end{array}$ & $\begin{array}{l}\text { Retrospective } \\
\text { case series }\end{array}$ & $\begin{array}{l}9 \\
5 \text { CPE } \\
4 \text { empyemas }\end{array}$ & $\begin{array}{l}100000-250000 \text { in } \\
100 \mathrm{ml} \text { NS daily; } \\
\text { clamped } 2-4 \mathrm{~h} ; \\
\text { patient rotation }\end{array}$ & $\begin{array}{l}\text { Increased drainage; } \\
\text { CXR improvment; no } \\
\text { further surgery }\end{array}$ & 44 & $\begin{array}{l}2 \text { fever } \\
1 \text { chest pain }\end{array}$ & $\begin{array}{l}\text { 1-3 instillations; } \\
\text { successful only in CPE }\end{array}$ \\
\hline $\begin{array}{l}\text { Willsie- } \\
\text { Ediger } \\
\end{array}$ & $\begin{array}{l}\text { Retrospective } \\
\text { case series }\end{array}$ & 3 empyemas & $\begin{array}{l}240000 \text { in } 100 \mathrm{ml} \\
\text { NS daily; clamped }\end{array}$ & $\begin{array}{l}\text { Increased drainage; } \\
\text { CXR improvement }\end{array}$ & 100 & None & $\begin{array}{l}\text { Failed chest tube } \\
\text { drainage; } 1-10 \\
\text { instillations }\end{array}$ \\
\hline $\begin{array}{l}\text { Aye } \\
(1991)^{36}\end{array}$ & $\begin{array}{l}\text { Retrospective } \\
\text { case series }\end{array}$ & 9 empyemas & $\begin{array}{l}250000 \text { in } 100 \mathrm{ml} \\
\text { NS daily; clamped } \\
4 \mathrm{~h}\end{array}$ & $\begin{array}{l}\text { Increased drainage; } \\
\text { CXR improvement: } \\
\text { resolution of fever }\end{array}$ & 89 & $\begin{array}{l}3 \text { temp to } \\
38.9^{\circ} \mathrm{C}, 2 \text { chest } \\
\text { wall erythema } \\
\text { \& erpmate }\end{array}$ & $\begin{array}{l}\text { Failed chest tube } \\
\text { drainage; } 1-4 \\
\text { instillations }\end{array}$ \\
\hline $\begin{array}{l}\text { Henke } \\
(1992)^{37}\end{array}$ & $\begin{array}{l}\text { Retrospective } \\
\text { case series }\end{array}$ & $12 \mathrm{CPE}$ & $\begin{array}{l}250000 \text { in } 30-60 \mathrm{ml} \\
\text { NS daily; clamped } \\
2 \mathrm{~h}\end{array}$ & $\begin{array}{l}\text { Increased drainage; } \\
\text { CXR improvement; } \\
\text { clinical improvement }\end{array}$ & 67 & $\begin{array}{l}\text { None } \\
\text { Nond }\end{array}$ & $\begin{array}{l}\text { Failed chest tube } \\
\text { drainage; } 1-7 \\
\text { instillations }\end{array}$ \\
\hline $\begin{array}{l}\text { Alfageme } \\
(1993)^{18}\end{array}$ & $\begin{array}{l}\text { Retrospective } \\
\text { case series }\end{array}$ & 8 empyemas & 250000 daily & $\begin{array}{l}\text { Complete CXR } \\
\text { improvement }\end{array}$ & 100 & None & $\begin{array}{l}\text { Failed chest tube } \\
\text { drainage; none }\end{array}$ \\
\hline $\begin{array}{l}\text { Rosen } \\
(1993)^{38}\end{array}$ & $\begin{array}{l}\text { Retrospective } \\
\text { case series }\end{array}$ & 5 empyemas & $\begin{array}{l}12300-136000 / \mathrm{kg} \\
\text { in } 50 \mathrm{ml} \text { NS daily; } \\
\text { clamped } 2 \mathrm{~h} \text {; rotate }\end{array}$ & Clinical improvement & 100 & 1 high fever & $\begin{array}{l}\text { Failed chest tube } \\
\text { drainage; } 2-5 \\
\text { instillations; }\end{array}$ \\
\hline $\begin{array}{l}\text { Bouros } \\
(1994)^{39}\end{array}$ & $\begin{array}{l}\text { Prospective case } \\
\text { series }\end{array}$ & $\begin{array}{l}20 \\
15 \text { CPE } \\
5 \text { empyemas }\end{array}$ & $\begin{array}{l}250000 \text { in } 100 \mathrm{ml} \\
\text { NS daily; clamped } \\
3 \mathrm{~h}\end{array}$ & $\begin{array}{l}\text { Clinical and } \\
\text { radiologic } \\
\text { improvement }\end{array}$ & 95 & 1 high fever & $\begin{array}{l}\text { Failed chest tube } \\
\text { drainage; } 3-10 \\
\text { instillations }\end{array}$ \\
\hline $\begin{array}{l}\text { Taylor } \\
(1994)^{40}\end{array}$ & $\begin{array}{l}\text { Retrospective } \\
\text { case series }\end{array}$ & 11 empyemas & $\begin{array}{l}250000 \text { in } 100 \mathrm{ml} \\
\text { NS daily; clamped } \\
4 \mathrm{~h}\end{array}$ & $\begin{array}{l}\text { Clinical outcome; } \\
\text { pleural drainage; us } \\
+ \text { CXR assessment }\end{array}$ & 73 & None & $\begin{array}{l}\text { Failed chest tube } \\
\text { drainage; 2-6 } \\
\text { instillations; 8-12 F } \\
\text { catheters }\end{array}$ \\
\hline $\begin{array}{l}\text { Chin } \\
(1996)^{41}\end{array}$ & $\begin{array}{l}\text { Prospective case } \\
\text { series }\end{array}$ & $\begin{array}{l}13 \text { CPE and } \\
\text { empyemas }\end{array}$ & $\begin{array}{l}250000 \text { in } 100 \mathrm{ml} \\
\text { NS daily; clamped } \\
4 \mathrm{~h}\end{array}$ & $\begin{array}{l}\text { Clinical and } \\
\text { radiological } \\
\text { resolution; pleural } \\
\text { drainage }\end{array}$ & 69 & None & $\begin{array}{l}\text { Failed chest tube } \\
\text { drainage; } 1-10 \\
\text { instillations }\end{array}$ \\
\hline $\begin{array}{l}\text { Jerjes- } \\
\text { Sanchez } \\
(1996)^{42}\end{array}$ & $\begin{array}{l}\text { Prospective } \\
\text { multicentre case } \\
\text { series }\end{array}$ & 30 empyemas & $\begin{array}{l}250000 \text { in } 100 \mathrm{ml} \\
\text { NS daily; clamped } \\
4 \text { h; patient } \\
\text { rotation }\end{array}$ & $\begin{array}{l}\text { Pleural drainage; CXR } \\
\text { improvement; pulm } \\
\text { function } \\
\text { improvement }\end{array}$ & 93 & $\begin{array}{l}2 \text { transient AMS } \\
3 \text { pleuritic pain } \\
1 \text { low grade } \\
\text { fever }\end{array}$ & $\begin{array}{l}\text { Failed chest tube } \\
\text { drainage; } 2-10 \\
\text { instillations }\end{array}$ \\
\hline $\begin{array}{l}\text { Laisaar } \\
(1996)^{43}\end{array}$ & $\begin{array}{l}\text { Retrospective } \\
\text { case series }\end{array}$ & $\begin{array}{l}22 \\
21 \text { empyemas } \\
1 \text { CPE }\end{array}$ & $\begin{array}{l}250000 \text { in } 100 \mathrm{ml} \\
\text { NS daily; clamped } \\
3 \mathrm{~h}\end{array}$ & $\begin{array}{l}\text { Increased pleural } \\
\text { drainage, CXR } \\
\text { improvement; clinical } \\
\text { imorovement }\end{array}$ & 68 & $\begin{array}{l}\text { Chest pain } \\
\text { fever } \\
\text { bleeding }\end{array}$ & $\begin{array}{l}\text { Failed chest tube } \\
\text { drainage; } 2-8 \\
\text { instillations }\end{array}$ \\
\hline $\begin{array}{l}\text { Roupie } \\
(1996)^{44}\end{array}$ & $\begin{array}{l}\text { Retrospective } \\
\text { case series }\end{array}$ & 16 empyemas & $\begin{array}{l}250000 \text { in } 30-50 \mathrm{ml} \\
\mathrm{NS} \text { daily; clamped } \\
2 \mathrm{~h} \text {; or via needle } \\
\text { under CT auidance }\end{array}$ & $\begin{array}{l}\text { Increased pleural } \\
\text { drainage; CT } \\
\text { improvement }\end{array}$ & 88 & $\begin{array}{l}1 \text { chills and } \\
\text { fever }\end{array}$ & $\begin{array}{l}\text { Failed chest tube } \\
\text { drainage; } 1-3 \\
\text { instillations }\end{array}$ \\
\hline $\begin{array}{l}\text { Chin } \\
(1977)^{45}\end{array}$ & $\begin{array}{l}\text { Prospective case } \\
\text { series; } \\
\text { sequential SK vs } \\
\text { tube }\end{array}$ & $\begin{array}{l}23 \\
17 \text { empyemas } \\
6 \mathrm{CPE}\end{array}$ & $\begin{array}{l}25000 \text { in } 100 \mathrm{ml} \\
\mathrm{NS} \text { daily; clamped } \\
4 \mathrm{~h}\end{array}$ & $\begin{array}{l}\text { Pleural drainage } \\
<50 \mathrm{ml} \text {; clinical \& } \\
\text { radiologic resolution }\end{array}$ & 78 & None & $1-10$ instillations \\
\hline $\begin{array}{l}\text { Bouros } \\
(1997)^{46}\end{array}$ & $\begin{array}{l}\text { tube } \\
\text { Prospective } \\
\text { randomised } \\
\text { double blind SK } \\
\text { vs UK }\end{array}$ & $\begin{array}{l}25 \\
20 \text { CPE } \\
5 \text { empyemas }\end{array}$ & $\begin{array}{l}250000 \text { in } 100 \mathrm{ml} \\
\text { NS daily; clamped } \\
3 \mathrm{~h}\end{array}$ & $\begin{array}{l}\text { Clinical outcome; } \\
\text { fluid drainage; CXR, } \\
\text { US, or CT } \\
\text { improvement }\end{array}$ & 92 & 2 high fever & $\begin{array}{l}\text { Failed chest tube } \\
\text { drainage; } 3-10 \\
\text { instillations }\end{array}$ \\
\hline $\begin{array}{l}\text { Davies } \\
(1997)^{47}\end{array}$ & $\begin{array}{l}\text { Randomised } \\
\text { controlled SK vs } \\
\text { saline }\end{array}$ & $\begin{array}{l}12 \\
7 \text { empyemas } \\
5 \text { CPE }\end{array}$ & $\begin{array}{l}250000 \text { in } 20 \mathrm{ml} \mathrm{NS} \\
\text { daily } \times 3\end{array}$ & $\begin{array}{l}\text { Pleural drainage; CXR } \\
\text { improvement }\end{array}$ & R100 & None & $\begin{array}{l}14 \mathrm{~F} \text { catheter; none } \\
\text { required surgery; } \\
\text { systemic fibrinolysis } \\
\text { or bleeding did not } \\
\text { occur }\end{array}$ \\
\hline $\begin{array}{l}\text { Wait } \\
(1997)^{48}\end{array}$ & $\begin{array}{l}\text { Prospective } \\
\text { randomised SK } \\
\text { vs VATS }\end{array}$ & $\begin{array}{l}9 \text { loculated } \\
\text { effusions or } \mathrm{pH} \\
\leq 7.20\end{array}$ & $\begin{array}{l}250000 \text { in } 100 \mathrm{ml} \\
\text { NS daily } \times 3 ; \\
\text { clamped } 3 \mathrm{~h}\end{array}$ & $\begin{array}{l}>50 \% \text { of original } \\
\text { fluid volume drained; } \\
\mathrm{T}<38^{\circ} \mathrm{C} ; \mathrm{WBC}<11 \mathrm{~K} ;\end{array}$ & 44 & Not reported & $\begin{array}{l}36 \mathrm{~F} \text { chest tube; } \mathrm{SK} \\
\text { failures salvaged with } \\
\text { VATS }\end{array}$ \\
\hline
\end{tabular}

patients in the control group and in none of the patients in the streptokinase group.

Chin and $\mathrm{Lim}^{45}$ reported on 52 patients (40 with empyema and 12 with complicated parapneumonic effusions) who were studied prospectively over a five year period (tables 1 and 3). The same criteria used to define empyema and complicated parapneumonic effusion were used as in the study by Davies and coleffusion were used as in the study by Davies and col-
leagues. In patients with large dependent pleural effuleagues. In patients with large dependent pleural effu-
sions, a $24 \mathrm{~F}$ chest tube was inserted at the bedside; in patients with multiloculated or non-dependent loculated effusions, a $7-12 \mathrm{~F}$ pigtail catheter was placed under ultrasound guidance. During the first 2.5 years of the study pleural space drainage alone was the treatment modality (29 patients) and during the last 2.5 years of the study streptokinase (23 patients) was used. The same physicians were responsible for patient care during the entire study with a consistent approach to initial empiric antibiotics, indications for further intervention, and eventual hospital discharge. Streptokinase was administered intrapleurally in a dose of $250000 \mathrm{U}$ in $100 \mathrm{ml}$ of normal saline daily with tube clamping for four hours. The total number of doses of streptokinase was determined by patient response. A significantly larger volume of pleural fluid was drained from patients in the streptokinase group (mean (SD) $2.0(1.5)$ l) than in the drainage group $(1.0(1.0) 1)$. There were no significant differences between the two treatment groups in time to defervescence, hospital stay, need for surgical intervention, or mortality. 


\begin{tabular}{|c|c|c|c|c|c|c|c|}
\hline Reference & $\begin{array}{l}\text { Study } \\
\text { design }\end{array}$ & $\begin{array}{l}\text { Number of } \\
\text { patients/ } \\
\text { diseases }\end{array}$ & $\begin{array}{l}\text { Urokinase } \\
\text { dose }(U)\end{array}$ & Success criteria & $\begin{array}{l}\text { Success } \\
(\%)\end{array}$ & $\begin{array}{l}\text { Complica- } \\
\text { tions }\end{array}$ & Comments \\
\hline $\begin{array}{l}\text { Moulton } \\
(1989)^{49}\end{array}$ & $\begin{array}{l}\text { Retrospective } \\
\text { case series }\end{array}$ & $\begin{array}{l}11 \\
8 \text { positive Gram } \\
\text { stain or culture } \\
3 \mathrm{pHH}<7.00\end{array}$ & $\begin{array}{l}80000-150000 \\
\text { several times daily, } \\
\text { clamped } 1-2 \mathrm{~h}\end{array}$ & $\begin{array}{l}\text { Clinical outcome; } \\
\text { absence of residual } \\
\text { fluid }\end{array}$ & $\begin{array}{l}91 \text { complete } \\
9 \text { partial }\end{array}$ & None & $\begin{array}{l}\text { Failed chest tube or } \\
\text { catheter drainage; 3-8 } \\
\text { instillations; } 12-14 \mathrm{~F} \\
\text { catheters }\end{array}$ \\
\hline $\begin{array}{l}\text { Lee } \\
(1991)^{50}\end{array}$ & $\begin{array}{l}\text { Prospective case } \\
\text { series }\end{array}$ & 10 empyemas & $\begin{array}{l}100000 \text { in } 100 \mathrm{ml} \\
\mathrm{D}_{5} \mathrm{~W} \text {; clamping }\end{array}$ & $\begin{array}{l}\text { Complete drainage } \\
\text { by CXR }\end{array}$ & 90 & None & $\begin{array}{l}8 \mathrm{~F} \text { catheters; } 1-7 \\
\text { instillations }\end{array}$ \\
\hline $\begin{array}{l}\text { Pollak } \\
(1994)^{51}\end{array}$ & $\begin{array}{l}\text { Retrospective } \\
\text { case series }\end{array}$ & $\begin{array}{l}5 \\
4 \text { CPE } \\
1 \text { empyema }\end{array}$ & $\begin{array}{l}180000-250000 \text { in } \\
100-500 \mathrm{ml} \mathrm{NS} \\
\text { daily; clamped } \\
30-180 \mathrm{~min} \text {; } \\
\text { rotated }\end{array}$ & $\begin{array}{l}\text { Complete drainage; } \\
\text { clinical } \\
\text { improvement }\end{array}$ & 80 & None & $\begin{array}{l}\text { Failed chest tube } \\
\text { drainage; } 1-2 \\
\text { instillations }\end{array}$ \\
\hline $\begin{array}{l}\begin{array}{l}\text { Robinson } \\
(1994)^{52}\end{array} \\
\text { lat }\end{array}$ & $\begin{array}{l}\text { Retrospective } \\
\text { case series }\end{array}$ & $\begin{array}{l}10 \mathrm{CPE} \text { or } \\
\text { empyemas }\end{array}$ & $\begin{array}{l}100000 \text { in } 100 \mathrm{ml} \\
\text { NS daily; clamped } \\
6-12 \mathrm{~h}\end{array}$ & $\begin{array}{l}\text { Resolution by CT; } \\
\text { clinical outcome }\end{array}$ & 80 & None & \multirow{5}{*}{$\begin{array}{l}\text { Two initially improved } \\
\text { but subsequently } \\
\text { required decortication; } \\
1-14 \text { instillations } \\
\text { Failed chest tube } \\
\text { drainage; } 10-12 \mathrm{~F} \\
\text { catheters, } 3-7 \\
\text { instillations } \\
\text { US with linear septations } \\
\text { and honeycombing } \\
\text { associated with failure; } \\
10-12 \mathrm{~F} \text { catheters } \\
\text { Children (1.5-12y) } \\
\text { failure of tube drainage } \\
\text { and large loculations; a } \\
\text { single UK failure due to } \\
\text { late treatment } \\
\text { Failure of CT drainage; } 2 \\
\text { UK patients required } \\
\text { surgery; } 3-12 \text { instillations }\end{array}$} \\
\hline $\begin{array}{l}\text { Bouros } \\
(1996)^{53}\end{array}$ & $\begin{array}{l}\text { Prospective case } \\
\text { series }\end{array}$ & $\begin{array}{l}20 \\
13 \text { CPE } \\
7 \text { empyemas }\end{array}$ & $\begin{array}{l}50000 \text { in } 100 \mathrm{ml} \mathrm{NS} \\
\text { daily; clamped } 3 \mathrm{~h}\end{array}$ & $\begin{array}{l}\text { Volume of fluid } \\
\text { drained; CXR; serial } \\
\text { US/CT }\end{array}$ & 95 & None & \\
\hline $\begin{array}{l}\text { Park } \\
(1996)^{54}\end{array}$ & $\begin{array}{l}\text { Retrospective } \\
\text { case series }\end{array}$ & 10 empyemas & $\begin{array}{l}\text { Total } 250000 \text { daily } \\
\text { in } 3 \text { doses; clamped } \\
1-2 \mathrm{~h}\end{array}$ & $\begin{array}{l}\text { Lung expansion on } \\
\text { CXR }\end{array}$ & $\begin{array}{l}60 \text { complete } \\
30 \text { partial }\end{array}$ & None & \\
\hline $\begin{array}{l}\text { Kornecki } \\
(1997)^{55}\end{array}$ & $\begin{array}{l}\text { Retrospective } \\
\text { case series }\end{array}$ & 5 empyemas & $\begin{array}{l}100000 \text { in } 100 \mathrm{ml} \\
\text { NS daily; clamped } \\
12 \mathrm{~h} \text {, suction } 12 \mathrm{~h}\end{array}$ & $\begin{array}{l}\text { US vol of daily fluid } \\
\text { drained }\end{array}$ & 80 & None & \\
\hline $\begin{array}{l}\text { Bouros } \\
(1997)^{46}\end{array}$ & $\begin{array}{l}\text { Prospective } \\
\text { randomized } \\
\text { double blind }\end{array}$ & $\begin{array}{l}25 \\
19 \text { CPE } \\
6 \text { empyema }\end{array}$ & $\begin{array}{l}100000 \text { in } 100 \mathrm{ml} \\
\text { NS daily; clamped } \\
3 \mathrm{~h}\end{array}$ & $\begin{array}{l}\text { Clinical outcome; } \\
\text { fluid drainage; } \\
\text { CXR; US and CT }\end{array}$ & 92 & None & \\
\hline
\end{tabular}

Table 3 Trials of streptokinase versus tube drainage, saline, VATS, and urokinase in complicated parapneumonic effusions Table 3 Trials of streptokinase vers
(CPE) and pneumonic empyemas

\begin{tabular}{|c|c|c|c|c|c|c|c|}
\hline Reference & $\begin{array}{l}\text { Study } \\
\text { design }\end{array}$ & $\begin{array}{l}\text { Number of } \\
\text { patients }\end{array}$ & $\begin{array}{l}\text { Dose of } S K \text { or UK } \\
\text { (U) }\end{array}$ & Results & & & Comments \\
\hline \multirow{2}{*}{$\begin{array}{l}\text { Chin } \\
(1977)^{45}\end{array}$} & \multirow{2}{*}{$\begin{array}{l}\text { Sequential } \\
\text { Consecutive } \\
\text { SK vs. tube } \\
\text { drainage }\end{array}$} & \multirow{2}{*}{$\begin{array}{l}52(29 \\
\text { drainage } \\
\text { only vs } 23 \\
\text { SK) }\end{array}$} & \multirow{2}{*}{$\begin{array}{l}\text { SK } 250000 \text { in } \\
100 \mathrm{mI} \text { NS daily } \\
\text { via } 24 \mathrm{~F} \text { chest } \\
\text { tube or } 7-12 \mathrm{~F} \\
\text { catheter; } \\
\text { Clamped } 4 \mathrm{~h}\end{array}$} & \multirow{3}{*}{$\begin{array}{l}\text { Hospital days } \\
\text { Time to afebri } \\
\text { Volume of flui } \\
\text { Surg (\%) } \\
\text { Mortality (\%) } \\
\text { Failure (\%) }\end{array}$} & SK & Tube & 40 empyema \\
\hline & & & & & $\begin{array}{l}22(11) \\
10.6(7) \\
2(1.5) \\
22 \\
9 \\
20\end{array}$ & $\begin{array}{c}\text { drainage } \\
21(12) \\
7.7(5) \\
1(1)^{*} \\
14 \\
24\end{array}$ & $\begin{array}{l}12 \text { CPE } \\
\text { Larger volume drained in SK } \\
\text { group; No differences in } \\
\text { duration of fever, hospital stay, } \\
\text { need for surgery, or mortality }\end{array}$ \\
\hline \multirow{5}{*}{$\begin{array}{l}\text { Davies } \\
(1997)^{47}\end{array}$} & \multirow{5}{*}{$\begin{array}{l}\text { Randomised } \\
\text { SK vs saline }\end{array}$} & \multirow{5}{*}{$\begin{array}{l}24(12 \\
\text { saline vs } \\
12 \text { SK) }\end{array}$} & \multirow{5}{*}{$\begin{array}{l}\text { SK } 250000 \text { in } \\
20 \mathrm{ml} \text { NS daily for } \\
3 \text { days; Clamped } \\
2 \mathrm{~h} \text { vs Control } \\
20 \mathrm{ml} \text { of saline } \\
\text { daily for } 3 \text { days }\end{array}$} & & $\mathrm{SK}^{30}$ & $\begin{array}{l}34 \\
\text { Tube } \\
\text { drain }\end{array}$ & \multirow{5}{*}{$\begin{array}{l}13 \text { empyema } \\
11 \text { CPE } \\
3 \text { saline patients required } \\
\text { surgery } \\
0 \text { SK patients required surgery }\end{array}$} \\
\hline & & & & \multirow{4}{*}{$\begin{array}{l}\text { Hospital days } \\
\text { Duration of drainage } \\
\text { (d) } \\
\text { Time to afebrile (d) } \\
\text { Vol of drainage } \\
\text { during Rx (mI) } \\
\text { Vol of drainage } \\
\text { overall (ml) } \\
\text { CXR improvement } \\
\text { (cm fluid collections) }\end{array}$} & $\begin{array}{c}16(13) \\
9(5)\end{array}$ & $\begin{array}{l}\text { dranagage } \\
13(8) \\
9(3)\end{array}$ & \\
\hline & & & & & $\begin{array}{c}6(6) \\
391(200)\end{array}$ & $\begin{array}{c}8(6) \\
124(267)^{*}\end{array}$ & \\
\hline & & & & & 2564 (1663) & $1059(502)^{*}$ & \\
\hline & & & & & $6(2.7)$ & $3.4(2.7)^{*}$ & \\
\hline $\begin{array}{l}\text { Wait } \\
(1977)^{48}\end{array}$ & $\begin{array}{l}\text { Randomised } \\
\text { SK vs VATS }\end{array}$ & $\begin{array}{l}20(11 \\
\text { VATS vs } 9 \\
\text { SK) }\end{array}$ & $\begin{array}{l}\text { SK } 250000 \text { in } \\
100 \mathrm{ml} N \mathrm{NS} \text { daily } \\
\text { for } 3 \text { days via } 36 \\
\text { F chest tube; } \\
\text { Clamped } 4 \mathrm{~h} \text { vs } \\
\text { Immediate VATS }\end{array}$ & \multirow{5}{*}{ 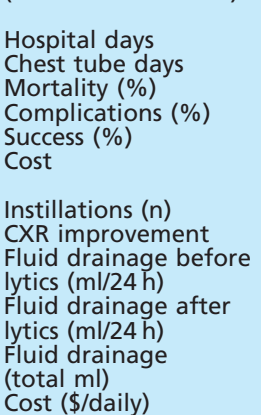 } & $\begin{array}{l}\text { SK } \\
12.1(1.1) \\
9.8(1.3) \\
11 \\
9 \\
44\end{array}$ & $\begin{array}{l}\text { VATS } \\
\quad 8.7(1.1)^{*} \\
5.8(1.1)^{*} \\
90 \\
91 * \\
91 *\end{array}$ & $\begin{array}{l}\text { Loculated effusion or } \leq 7.20 \\
\text { Failure in SK group determined } \\
\text { at } 3 \text { days by } 250 \% \text { of fluid } \\
\text { drained, temp } \geq 38^{\circ} \mathrm{C} \text {, and WBC } \\
>11000 / \mu 1\end{array}$ \\
\hline \multirow[t]{4}{*}{$\begin{array}{l}\text { Bouros } \\
(1997)^{46}\end{array}$} & \multirow[t]{4}{*}{$\begin{array}{l}\text { Randomised } \\
\text { SK vs UK }\end{array}$} & \multirow[t]{4}{*}{$\begin{array}{l}50 \text { ( } 25 \text { UK } \\
\text { vs } 25 \mathrm{SK})\end{array}$} & \multirow{4}{*}{$\begin{array}{l}\text { SK } 250000 \text { in } \\
100 \mathrm{ml} \mathrm{NS} \text { daily vs } \\
\text { UK } 10000 \text { in } \\
100 \mathrm{ml} \text { NS daily; } \\
\text { Clamped } 3 \mathrm{~h} \text {. } \\
\text { Re-instilled in } \\
\text { persistent } \\
\text { effusion and } \\
\text { drainage }<50 \mathrm{ml} / \\
24 \mathrm{~h}\end{array}$} & & $\begin{array}{l}\text { SK }{ }^{16 K} \\
6(2.2) \\
2.5(0.7) \\
48(23)\end{array}$ & $\begin{array}{l}\mathrm{UK}^{24 \mathrm{~K}} \\
5.9(2.1) \\
2.7(0.8) \\
56(31)\end{array}$ & \multirow{4}{*}{$\begin{array}{l}\text { SK } 20 \text { CPE and } 5 \text { empyemas } \\
\text { UK } 19 \text { CPE and } 6 \text { empyemas } \\
\text { SK } 7 \text { transient fever; } 2 \text { with } \\
\text { temp } 39-40{ }^{\circ} \mathrm{C} \\
\text { SK } 2 \text { failures requiring surgery } \\
\text { UK } 2 \text { failures requiring surgery }\end{array}$} \\
\hline & & & & & 380 (99) & $421(110)$ & \\
\hline & & & & & $1596(68)$ & $1510(55)$ & \\
\hline & & & & & 122 & 212 & \\
\hline
\end{tabular}

A small randomised trial compared chest tube drainage with intrapleural streptokinase and video-assisted thoracoscopic surgery (VATS) for the treatment of complicated effusions or parapneumonic empyemas ${ }^{48}$ (tables
1 and 3). Eligible patients had to have either loculated pleural effusions or a pleural fluid $\mathrm{pH}$ of $<7.20$. Twelve of the 20 patients had positive bacterial cultures, most of which were anaerobic or microaerophilic organisms. 
Patients were randomised to receive either chest tube drainage with streptokinase $(n=9)$ or VATS $(n=11)$. Patients randomised to tube thoracostomy with streptokinase had a $36 \mathrm{~F}$ chest tube placed under the supervision of a pulmonary fellow or attending and appropriate placemet appropriate placentiophy. was instilled intrapleurally and the tube clamped for was instilled intrapleurally and the tube clamped for
four hours. Each patient received three daily doses of four hours. Each patient received three daily doses of streptokinase. Following the last dose of streptokinase lowing criteria: adequate drainage was defined as the resolution of $>50 \%$ of the original volume of pleural fluid on chest radi $<38^{\circ} \mathrm{C}$; and a peripheral leucocyte count of $<11000 / \mu \mathrm{l}$ The streptokinase failures were referred to thoracic surgery for additional treatment. The streptokinase successes were followed until chest drainage was $<100 \mathrm{~m}$ per day, at which time tubes were removed and the patient discharged. Patients randomised to VATS wen immediately to surgery without prior chest tube placement.

Based on the study criteria, the VATS group had a significantly higher primary treatment success, fewer days on chest tube drainage, and shorter hospital stays. There was a trend for a lower hospital cost in the VATS group. All streptokinase treatment failures were salvaged by VATS and none required thoracotomy. The problems with this study are the small sample size, the question of adequate chest tube placement by house officers at the bedside, uncertainty of chest tube position judged only by standard chest radiography, the short duration of streptokinase treatment, and the strict radiographic criteria for success. It is possible that more frequent or prolonged use of streptokinase would have resulted in a better outcome. It appears that the "deck was stacked" against the streptokinase group because of the aforementioned problems. The cost trend was greater fo the streptokinase group because of the addition of the five VATS procedures.

A randomised study compared the efficacy, safety profile, and cost of two fibrinolytic agents in the treatment of complicated parapneumonic effusions. Bouros and co-workers ${ }^{46}$ studied 50 consecutive patients with complicated parapneumonic effusion ${ }^{39}$ or empyema ${ }^{11}$ who were randomly allocated to receive either streptokinase (25 patients) or urokinase in a double blind fashion (tables 1,2 and 3). Criteria for inclusion included multiloculated complicated parapneumonic effusions or loculated empyemas confirmed by CT scanning, ultrasound, or both and failure of drainage via tube thoracostomy $(<70 \mathrm{ml}$ during the previous 24 hours).

All patients had pleural space drainage with a 28 to $32 \mathrm{~F}$ chest tube. Twenty five patients received 250000 $\mathrm{U}$ streptokinase in $100 \mathrm{ml}$ of normal saline and 25 received urokinase $100000 \mathrm{U}$ in $100 \mathrm{ml}$ normal saline. Chest tubes in both groups were clamped for three hours following instillation and re-opened to suction. Streptokinase or urokinase was re-instilled if, on ultrasound or CT scanning, a persistent pleural effusion was seen and the amount of pleural fluid drainage was $<50 \mathrm{ml}$ during the previous 24 hours. Clinical and radiological improvement was noted in all but two patients in each group who required surgical intervention. Mean (SD) instillations were $6(2.7)$ in the streptokinase group and $5.9(2.1)$ in the urokinase group. The chest radiographic improvement score was the same in both groups. The mean volume of pleural fluid drained during the firs 24 hours after instillation was significantly increased in both groups compared with the volume drained in the
24 hours prior to instillation. A significant increase in the mean daily pleural fluid drainage was seen after either drug instillation compared with the volume drained in the 24 hours prior to treatment; however, there was no difference between streptokinase and urokinase. High fever $\left(39-40^{\circ} \mathrm{C}\right)$ was seen in th he patients on twice that of streptokinase, with the mean hospital stay being about 10 days in both groups. In this comparative study both fibrinolytic agents were effective adjuncts in the management of parapneumonic effusions.

\section{Intrapleural streptokinase in experimental} empyema

Strange and colleagues ${ }^{56}$ studied the effect of intrapleural streptokinase compared to saline control in a rabbit model of empyema. Streptokinase or saline was instilled daily for a total of three days immediately after bacterial inoculation of the pleural space. At day 4 following bacterial inoculation the streptokinase treated animals had a significantly increased amount of pleura fluid and fewer pleural adhesions than the saline treated control animals but comparable amounts of visceral and parietal pleural thickening. No evidence of systemic fibrinolysis was observed one hour after intrapleural streptokinase instillation. The investigators attempted to prevent fibrin deposition before a pleural peel was established, yet failed to completely prevent pleural adhesion formation, although the number of adhesions was significantly less than in the control group.

A possible explanation for the failure to completely prevent pleural adhesions is the rapid binding of streptokinase within an inflamed pleural space. While the half life of streptokinase intravascularly is $15-30$ minutes, ${ }^{57}$ it is probably shorter in the inflammatory pleural space. Streptokinase normally binds to plasminogen and the Streptified plasminogen co verted to plasmin. The proteolytic activity of plasmin on fibrin, however, can be blocked by a number of protease inhibitors such as $\alpha_{2}$-macroglobulin and $\alpha_{2}$ antiplasmin found in high concentrations in the inflamed pleural space. ${ }^{14}$ The increased procoagulant activity and high concentrations of plasminogen activator inhibitor 1 and plasminogen activator inhibitor 2 , which have fluid, ${ }^{14}$ may explain the progressive fibrin deposition that can occur between 24 hour streptokinase dosing. It is possible that an increased frequency of dosing would increase the success of fibrinolytic agents.

It is unclear, in the experimental study, why streptokinase produced an increased volume of pleural fluid. Streptokinase alone did not produce demonstrable effusions while streptokinase, in the setting of a noninfected inflammatory pleurisy, did produce large effusions. The possibility that pleural fibrinolysis resulted in an increased volume of inflammatory fluid entering the pleural space from the visceral or parietal pleura while not affecting the patency of the pleural stoma might explain these findings.

\section{Systemic fibrinolytic activity of intrapleural} streptokinase

The most comprehensive study of the systemic fibrinolytic effect of intrapleural streptokinase was recently published by Davies and colleagues ${ }^{58}$ who studied the systemic fibrinolytic activity of two intrapleural streptokinase regimens. Eight patients received a single dose of $250000 \mathrm{U}$ of intrapleural streptokinase and an ad- 
ditional eight received serial doses of $250000 \mathrm{U}$ every 12 hours for three days (total dose 1.5 million units). Each patient had the streptokinase retained in the pleural cavity for two hours. Prothrombin time, activated partia thromboplastin time, thrombin time, fibrinogen and Dwere measured before intrapleural administration of streptokinase. These end points were then re-measured 24 hours after streptokinase in the single dose group and at 24,48 and 72 hours in the group receiving serial doses. There were no physiological or statistical differences in any of the indices after administration of intrapleural streptokinase. Intrapleural streptokinase, even in a dose of 1.5 million units, did not cause significant activation of the systemic fibrinolytic system.

\section{Adverse effects of fibrinolytics}

Streptokinase has been reported to be antigenic and leads to antibody formation. Re-exposing patients to these agents may cause hypersensitivity or anaphylactoid reactions. These would be more likely to occur following intravenous administration but could conceivably occur following intrapleural administration. Lee and colleagues $^{59}$ found that antibodies to streptokinase were raised from four days to at least 54 months following the initial administration of streptokinase intravenously. The potential for serious adverse effects would therefore exist following re-exposure to streptokinase during this time period.

Major haemorrhage has been reported in a 36 year old man following instillation of $500000 \mathrm{U}$ of streptokinase intrapleurally and clamping of the chest tube for six hours. ${ }^{60}$ This patient drained $1500 \mathrm{ml}$ of bloody fluid, developed haematuria, gastrointestinal bleeding, pulmonary haemorrhage and epistaxis, in addition to oozing from puncture sites. Eleven hours after streptokinase \% $14 \%$ greater than before streptokinase. A single patient developed severe pleural space haemorrhage following intrapleural streptokinase (exact dose not recorded) one month following rib fractures. ${ }^{61}$

Frye and colleagues ${ }^{62}$ reported acute hypoxic respiratory failure following both streptokinase and urokinase given 24 hours apart for treatment of a loculated haemothorax. The streptokinase dose was $250000 \mathrm{U}$ and the urokinase dose was $450000 \mathrm{U}$. It is possible that the patient's severe hypoxaemia resulted from the effect of the products of fibrinolysis on the pulmonary circulation leading to a capillary leak pulmonary oedema. Alfageme and Vaquez ${ }^{63}$ reported a patient who (interapleural urokinase. Mild anaphylactoid reactions after intravenous urokinase have been reported but occasionally these reactions may be severe. ${ }^{645}$ Urokinase has not been found to be antigenic but urokinase can directly stimulate mast cells and basophils to release histamine and serotonin, causing vasodilatation and vascular permeability which can lead to hypotension and shock ${ }^{64}$ olved in the enhanced genicity seen during experimental anaphylaxis by decreasing the threshold for ventricular fibrillation and could have resulted from an anaphylactoid reaction. It is possible that a high single dose and prolonged dwell time might increase the risk of excess systemic absorption and systemic fibrinolysis. It would appear prudent not to use a single dose of streptokinase of $>250000$ U or a single dose of urokinase of $>100000 \mathrm{U}$ and to keep dwell hours. It may also be advisable to pretreat patients with acetaminophen and diphenhydramine prior to intrapleural fibrinolytics as has been recommended prior to intravenous urokinase. ${ }^{6}$

\section{Conclusion}

A single randomised controlled trial of intrapleural streptokinase has shown that pleural drainage was enhanced, the chest radiograph was improved, and surgery was avoided with streptokinase without causing local haemorrhage or systemic fibrinolysis. Intrapleural streptokinase and urokinase are equally efficacious in treating complicated parapneumonic effusions and empyemas complicated parapneumonic effusions and empyemas e surgical procedures. Streptokinase can be associated with high fever and allergic reactions, while urokinase has rarely been associated with severe adverse effects. However, urokinase is approximately twice the cost of streptokinase. There has not been a randomised trial of intrapleural fibrinolytic therapy versus VATS with an adequate number of patients. A large multicentre trial

\section{LEARNING POINTS}

* Fibrinolytic agents are a useful adjunct in the management of complicated parapneumonic effusions.

* Both streptokinase and urokinase are equally effective.

* Systemic fibrinolysis and bleeding complications are rare events.

* In the only randomised controlled trial, in which streptokinase was compared with saline control, the streptokinase group showed an increased volume of pleural fluid drainage during the treatment phase and, overall, an improved chest radiograph and no need for surgery.

* Intrapleural fibrinolytics, if used early in the fibrinopurulent stage of a parapneumonic effusion, may obviate the need for VATS or thoracotomy.

* A large multicentre randomised trial comparing a fibrinolytic and VATS early in the fibrinopurulent stage of a parapneumonic effusion is needed to determine the optimal management of these patients. 
with a meticulous study design comparing these modalities is needed.

1 Vital and Health Statistics. Current estimates from the National Health Interviezw Survey, 1991. DHHS Publication no. PHS 93-1512. Hyatts-
ville, MD: US Dept of Health and Human Services, December 1992. 2 Light RW, Girard WM, Jenkinson SG, et al. Parapneumonic effusions.

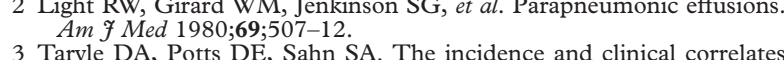
3 Tarle DA, Potts DE, Sahn SA. The incidence and clinical correlates
in parapneumonic effusions in pneumococcal pneumonia. Chest 1978; in parapne.
74:170-3.
Bartlett JG,

4 Bartlett JG, Finegold SM. Anaerobic infections of the lung and pleural space. Am Rev Respir Dis 1974;110:56-77.

pneumonic effusions. An analysis of physician practice patterns. Arch
prict pneumonic effusions.
Surg 1995; $130: 433-8$.

6 Ashbaugh DG. Empyema thoracis. Factors influencing morbidity and mortality. Chest 1991;99:1162-5.

Rev Respir Dis 1993;148:813-7. Strange C, Sahn SA. The clinician's perspective on parapneumonic 9 Wang NS. The preformed stomas connecting the pleural cavity and the 10 lymphatics in the parietal pleura. Am Rev Respir Dis 1975;111:12-20. effusions. Chest 1976;7:328-31.
11 Potts DE, Taryle DA, Sahn SA. The glucose-pH relationship in parapneumonic effusions. Arch Intern Med 1978; 138: 1378-80.
pahn SA, Taryle DA, Good JT Jr. Experimental empyema: time course and pathogenesis of pleural fluid acidosis and low pleural fluid glucose. A Am RA, Reller LB, Taryle DA, et al. The contribution of leukocytes
and bacteria to the low $\mathrm{pH}$ of empyema fluid. Am Rev Respir Dis

14 Idell S, Girard W, Koenig KB, et al. Abnormalities of pathways of fibrin

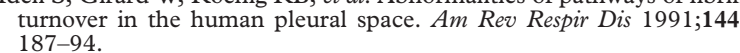
187-94.
Strange C, Tomlinson JR, Wilson C, et al. The pathology of experimental pleural injury with tetracycline, empyema, and carrageenan. Exp Mol
Pathol 1989;51:205-19.
6 Lew PD, Zublar R, Vaudaux P, et al. Decreased heat-labile opsonic activity and complement levels associated with evidence of C3 break17 Poe RH, Marin MG, Israel RH, et al. Utility of pleural fluid analysis in predicting tube thoracostor

18 Alfageme I, Munoz F, Pena N, et al. Empyema of the thorax in adults. Etiology, microbiologic findings and management. Chest 1993;103 19 Ferguson AD, Prescott RJ, Selkon JB, et al. The clinical course and

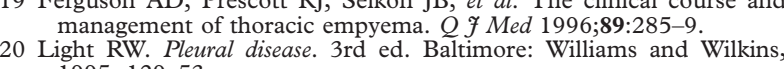
21 Braman SS, Donat WE. Explosive pleuritis: manifestation of group A
$\beta$-hemolytic streptococcal infection. Am ¥ Med 1986;81:723-6.

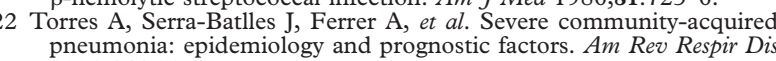
pneumonia: epicis

23 Himmelman RB, Callen PW. The prognostic value of loculations in parapneumonic pleural effusions. Chest 1986;90:852-6.
Stark DD, Federle

and empyema: radiography and computed tomography $A \Im R$ 1983, 141:163-7.
Aquino SL, Webb WR, Gushiken BJ. Pleural exudates and transudates: 25 Aquino SL, Webb WR, Gushiken BJ. Pleural exudates and transudates 26 Yang P-C, Luh K-H, Chang D-B, et al. Value of sonography in determining the nature of pleural effusion: analysis of 320 cases. $A F R$
1992;159:29-33. 27 McCloud TC, Flower CDR. Imaging the pleura: sonography, CT, and
MR imaging. AfR 1991;156:1145-53.
28 Light RW, MacGregor MI, Ball WC Jr, et al. Diagnostic significance of 29 Good JT Jr, Taryle DA, Maulitz RM, et al. The diagnostic value of

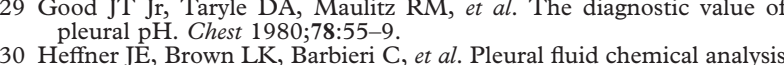
in parapneumonic effusions. A meta-analysis. Am f Respir Crit Care

31 Tillett WS, Sherry S. The effect in patients with streptococcal fibrinolysis (streptokinase) and streptococcal desoxyribonuclease on fibrinous,
purulent, and sanguinous pleural exudations. I Clin Invest 1949;28

32 Bergh NP, Ekroth R, Larsson S, et al. Intrapleural streptokinase in the treatment of haemothorax and empyema. Scand $\mathcal{F}$ Cardiovasc Surg
1977;11:265-8. 33 Lysy Y, Gavish A, Lieberson A, et al. Intrapleural instillation of strep- tokinase in the treatment of organizing empyema. Is 7 Med Sci 1989; tokinase in the treatment of organizing empyema. Is $f$ Med Sci 1989 ;
25:7. Mitchell ME, Alberts WM, Chandler KW, et al. Intrapleural strep-
tokinase in management of parapneumonic effusions. F Fla Med Assoc

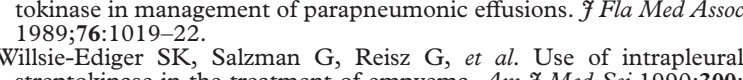
streptokinase in the treatment of empyema. Am $\mathcal{F}$ Med Sci 1990;300 streptokinas

36 Iaye RW, Froese DP, Hill LD. Use of purified streptokinase in empyema and hemothorax. Am $\mathcal{F}$ Surg 1991;161:560-2.
37 Henke CA, Leatherman JW. Intrapleurally administered streptokinase in the treatment of acute loculated non-purulent parapneumonic Rosen $\mathrm{H}$, Nadkarni V, Therou $\mathrm{M}$, et al. Intrapleural streptokinase as
adjunctive treatment for persistent empyema in pediatric patients. Chest 1993;103:11 90-3.
Bouros D, Schiza S, Panagou P, et al. Role of streptokinase in the treatment of acute loculated parapneumonic pleural effusions in empyema. Thorax 1994;49:852-5.
Taylor RFH, Reubens MB, Pearson MC, et al. Intrapleural streptokinase in the management of empyema. Thorax 1994;49:856

and pleural empyema: a four-year prospective study. Singapore Med $\mathscr{J}$

1996;37:631-5.
2 Jeries-Sanchez C, Ramirez-Rivera A, Elizalde JJ, et al. Intrapleural
fbrinolysis with with streptokinase as an adjunctive treatment in hemo43 Laisaar T, Tuttsepp E. Laisaar V. Early administration of intrapleural

pleural empyemas. Thorac Cardiovasc Surg 1996;44:252-6.
44 Roupie E, Bouabdallah K, Delclaux C, et al. Intrapleural administration of streptokinase in complicated purulent pleural effusion: a CT-guided 45 Chin NK, Lim TK. Controlled trial of intrapleural streptokinase in the
treatment of empyema and complicated parapneumonic effusions. Chest 1997;111:275-9.
Bouros D, Schiza S, Patsourakis P, et al. Intrapleural streptokinase versus urokinase in the treatment of complicated parapneumonic effusions. Am I Respir Crit Care Med 1997;155:291-5.
Davies RJO, Traill ZC, Gleeson FV. Randomized, controlled trial of Davies RJO, Traill ZC, Gleeson FV. Randomized, controlled trial of
intrapleural streptokinase in community-acquired pleural infection. intrapleural streptokinase
Thorax 1997;52:416-21.

48 Wait MA, Sharma S, Hohn J, et al. A randomized trial of empyema therapy. Chest 1997;111:1548-51.
49 Moulton JS, Moore PT, Mencini RA. Treatment of loculated pleural effusions with transcatheter intracavitary urokinase. $A \Im R$ 1989;153: 941-5. Im J-G, Kim YH, Hwang SH, et al. Treatment of thoracic

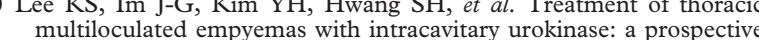
multiloculated empyemas with
studiogy $1991 ; 179$ : $771-5$.

51 Pollak JS, Passik CS. Intrapleural urokinase in the treatment of loculated pleural effusions. Chest 1994;105:868-73.
52 Robinson LA, Moulton AL, Fleming WH, et al. Intrapleural fibrinolytic treatment of multiloculated thoracic empyemas. Ann Thorac Surg 1994;57:803-14.
Bouros D, Schiza S, Tzanakis N, et al. Intrapleural urokinase in the
treatment of complicated parapneumonic pleural effusions in empyema. Eur Respir f 1996;9:1656-9.
54 Park CS, Chung WM, Lim MK, et al. Transcatheter instillation of
urokinase into loculated pleural effusion: analysis of treatment effect. urokinase into loculated pleural effusion: analysis of treatment effect.
$A \ngtr R$ 1966;167:649-52. 55 Kornecki A, Sivan Y. Treatment of a loculated pleural effusion with
intal 56 Strange C, Allen ML, Harley R, et al. Intrapleural streptokinase in experimental empyema. Am Rev Respir Dis 1993;147:962-6.
57 Pfeiter GW, Doerr F, Brod KH. Zur pharrakokinetik von ${ }^{131}$ - -strep-
tokinase an menschen. Klin Wochenschr 1969;47:482-6. 58 Davies CWH, Lok S, Davies ROS. The systemic fibrinolytic activity 328-30. 59 Lee HS, Cross S, Davidson R, et al. Raised levels of antistreptokinase
antibody and neutralization titres from four days to fifty-four months antibody and neutralization titres from four days to fifty-four months 14:84-9.
Godley PJ, Bell RC. Major hemorrhage following administration of 60 Godley PJ, Bell RC. Major hemorrhage following administration of
intrapleural streptokinase. Chest 1984;86:486-7. intrapleural streptokinase. Chest 1984;86:486-7.
61 Temes RT, Follis F, Kessler RM, et al. Intrapleural fibrinolytics in management of empyema thoracis. Chest 1996;110:102-6.
62 Frye MD, Jarratt M, Sahn SA. Acute hypoxemic respiratory failure 63 Alfageme I, Vazquez R. Ventricular fibrillation after intrapleural uro64 kerri JA, Intensive Care Med 1997;23:352. 64 Perri JA, Stahfeld KR, Villella ER, et al. The management of anaphylactoid reactions to urokinase. 7 Vasc Surg 1994;28:2846-7.
Vidovich RR, Heiselman DE, Hudock D. Treatment of urokinase-
related anaphylactoid reaction with intravenous famotidine. Ann Phar- 